\title{
Phonon Dynamics and Transport Properties of Copper Thiocyanate and Copper Selenocyanate Pseudo-halides
}

Nirpendra Singh ${ }^{1,2^{*}}$, Dalaver Anjum ${ }^{1}$, Gobind Das ${ }^{1}$, Issam Qattan ${ }^{1}$, Shashikant Patole $^{1}$, and Muhammad Sajjad ${ }^{3}$

${ }^{1}$ Department of Physics, Khalifa University of Science and Technology, PO BOX 127788, Abu Dhabi, United Arab Emirates (UAE)

${ }^{2}$ Center for Catalysis and Separation (CeCaS), Khalifa University of Science and Technology, PO BOX 127788, Abu Dhabi, United Arab Emirates (UAE)

${ }^{3}$ Applied Physics, Division of Materials Science, Department of Engineering Sciences and Mathematics, Lulea University of Technology, SE-97187 Lulea, Sweden

Corresponding Author: Nirpendra Singh

E-mail: Nirpendra.Singh@ku.ac.ae 

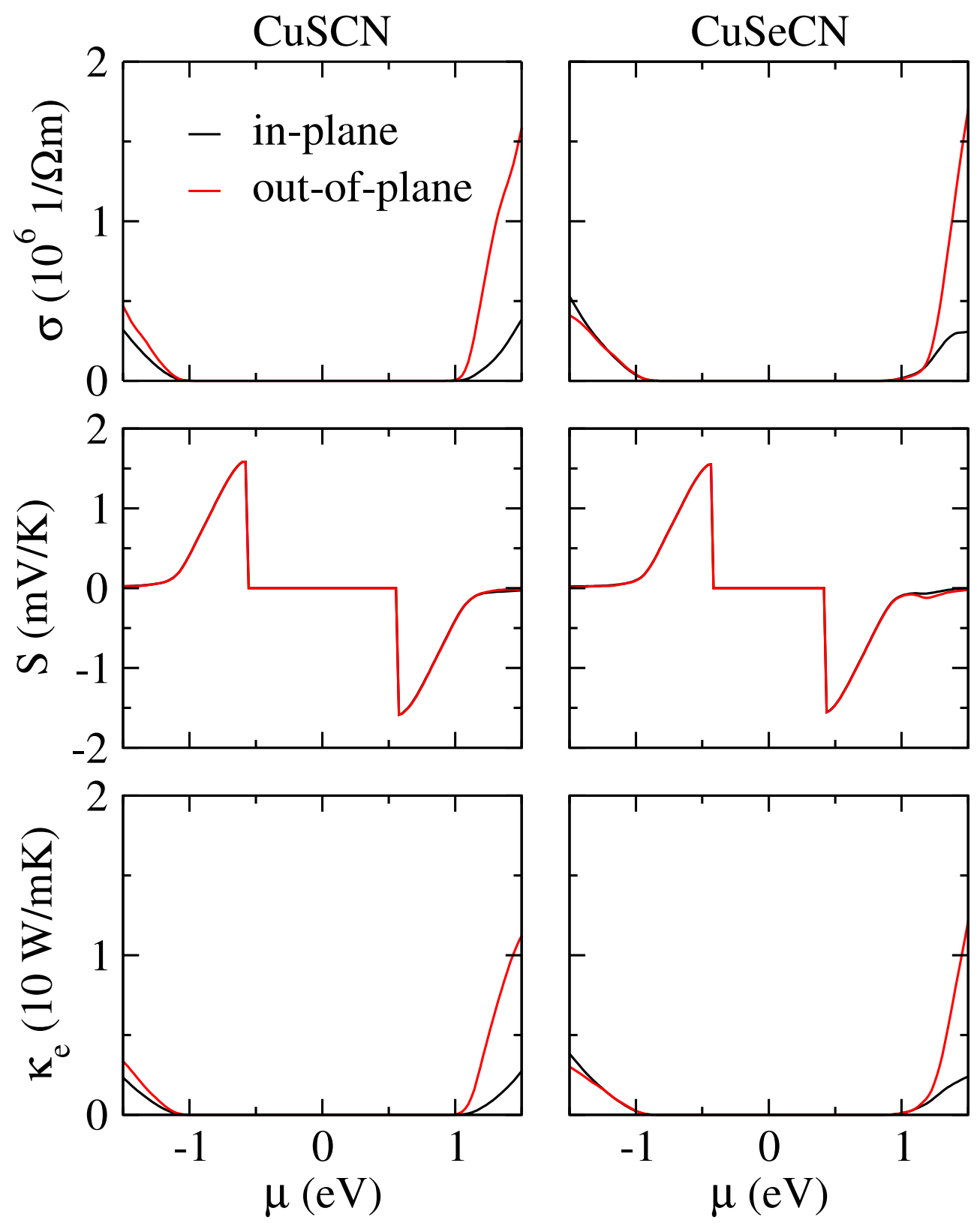

Figure S1. The calculated electrical conductivity $(\sigma)$, Seebeck coefficient (S), electronic thermal conductivity $\left(\kappa_{\mathrm{e}}\right)$ of $\mathrm{CuSCN}$ and $\mathrm{CuSeCN}$ as a function of chemical potential $(\mu)$, at $300 \mathrm{~K}$, along the in-plane and out-of-plane directions. 